\title{
Space Targets Classification and Recognition Based on Affine Invariant Moments
}

\author{
Wang Feng \\ Institute of Information Science \& Technology of \\ Zhengzhou \\ Zhengzhou 450002, China
}

\author{
Meng Fankun \\ Institute of Information Science \& Technology of \\ Zhengzhou \\ Zhengzhou 450002, China
}

\author{
Ma Debao \\ Institute of Information Science \& Technology of Zhengzhou \\ Zhengzhou 450002, China
}

\begin{abstract}
An affine transformation model is established for ground-based photoelectric imaging and detecting systems. This model shows that affine transform consists of five basic transformations, which are translation, scale, slant and rotation transformations. Six affine invariant moments are given with geometrical moments. Furthermore. affine invariant moment vectors of training data are calculated for four-class simulated space target images. For different viewpoint angle target images, the affine invariant moments are calculated. Simulation experiments are performed for space targets classification and recognition through their eigenvectors comparing. Simulated results show that the method of target images classification recognition based on affine invariant moments is effective.
\end{abstract}

Keywords-Space targets, Affine transform model, Affine invariant moments, Classification, Recognition

\section{INTRODUCTION}

With rapid development of space technology, countries in the world are relying more and more on space resources. It is very significant for national security and space engineering that how to carry out space targets(such as satellites, debrises etc.) surveillance and recognition efficiently. Ground-based high-resolution imaging systems can get gray sequence images at certain angle of view which are main means for space targets surveillance missions. Characteristic moment invariants of targets are constructed based on image gray distribution and these moment invariants reflect target information such as shape, size and so on, by which targets can be classified and recognized. The moment invariant is a classic method in recent 30 years for targets recognition problems in image processing. In 1962, Hu used the algebraic invariants theory, introduced the moment invariant theory into pattern recognition and inducted seven invariant moments[1], according to which effective recognition for printing characters was implemented. Presently, moment invariant methods are widely applied in target recognition, shape analysis and image analysis[2]. Based on space target gray sequential images, this paper establishes affine transform model and affine invariant moments are constructed.
Simulations for space targets classification and recognition validate the effectiveness of affine invariant moments.

\section{AN AFFINE TRANSFORM MODEL AND CONSTRUCTION}

\section{OF AFFINE INVARIANT MOMENTS}

If the size of a target in light axis direction is much shorter than the distance between the center of target and the camera, the imaging can be considered as linear affine transformation. When a ground-based photoelectric telescope detects space targets, the distance between targets and the detector arrives at hundreds of kilometers. This imaging process is satisfied with the affine transformation model. Affine invariants of targets can be used to recognize target images, which are constructed on condition of different affine transformation.

\section{A. Definition of geometric moments [3]}

Let $f(x, y)$ be a two-dimensional continuous image function, then its $(p+q)$-order origin moment can be defined in an oxy plane an follows:

$$
m_{p q}=\int_{-\infty}^{+\infty} \int_{-\infty}^{+\infty}\left[x^{p} y^{q} f(x, y)\right] d x d y \quad p, q=0,1,2, \cdots
$$

It is obvious that $m_{p q}$ is the projection on polynomials of $f(x, y)$, but $m_{p q}$ is related with $x-y$ coordinates. We define the $(p+q)$-order central moment as:

$u_{p q}=\int_{-\infty}^{+\infty} \int_{-\infty}^{+\infty}\left[(x-\bar{x})^{p}(y-\bar{y})^{q} f(x, y)\right] d x d y \quad p, q=0,1,2, \cdots$

In formula (2), $\bar{x}=m_{10} / m_{00}, \bar{y}=m_{01} / m_{00}$ and $(\bar{x}, \bar{y})$ represents the centroid of the gray-scale image. Thus, $u_{p q}$ is invariable to the translation transform, which reflects the gray distribution of target images. Supposing that $f(x, y)$ be the gray distribution of the target region $D$,where $(x, y) \in D$, and the gray distribution of the outside is regarded as zero, then the formula (1) and (2) can be obtained as follows: 


$$
\begin{gathered}
m_{p q}=\iint_{D}\left[x^{p} y^{q} f(x, y)\right] d x d y \quad p, q=0,1,2, \cdots \\
u_{p q}=\iint_{D}\left[(x-\bar{x})^{p}(y-\bar{y})^{q} f(x, y)\right] d x d y \quad p, q=0,1,2, \cdots
\end{gathered}
$$

As for the digital gray-scale image $f(i, j)$, the $(p+q)$-order origin moment and central moment are respectively defined as:

$$
\begin{aligned}
& m_{p q}=\sum_{i} \sum_{j} i^{p} j^{q} f(i, j) \quad p, q=0,1,2, \cdots \\
& u_{p q}=\sum_{i} \sum_{j}(i-\bar{i})^{p}(j-\bar{j})^{q} f(i, j) \quad p, q=0,1,2, \cdots
\end{aligned}
$$

In formula $(6),(\bar{i}, \bar{j})$ represents the centroid of the digital gray-scale image.

\section{B. Description of affine transformation model[2] [3] [4]}

Affine transformation is an important linear transformation and the corresponding model is described as follows. Supposing there be a point $P$ in the $o-x y$ plane, whose coordinate is $(x, y)$, and the corresponding point after affine transform is $P^{\prime}$, whose coordinate is $\left(x^{\prime}, y^{\prime}\right)$, then the two-dimensional affine transform model can be represented as:

$$
\left[\begin{array}{l}
x^{\prime} \\
y^{\prime}
\end{array}\right]=A\left[\begin{array}{l}
x \\
y
\end{array}\right]+\left[\begin{array}{l}
b_{1} \\
b_{2}
\end{array}\right]
$$

In formula (7), $A=\left[\begin{array}{ll}a_{11} & a_{12} \\ a_{21} & a_{22}\end{array}\right]$ is an affine transform matrix, which is satisfied with the nonsingular condition, i.e. $\operatorname{det}(A) \neq 0$. In two-dimensional space, the matrix $\mathrm{A}$ is decomposed into the following four single-parameter linear transformation. $\left[\begin{array}{l}b_{1} \\ b_{2}\end{array}\right]$ is translation parameter.

The scale transformation can be represented as two matrices:

$$
\begin{aligned}
& A_{s}=\left[\begin{array}{ll}
s & 0 \\
0 & s
\end{array}\right], s \geq 0 \\
& A_{t}=\left[\begin{array}{ll}
1 & 0 \\
0 & t
\end{array}\right]
\end{aligned}
$$

The slant transformation follows as:

$$
A_{n}=\left[\begin{array}{ll}
1 & n \\
0 & 1
\end{array}\right]
$$

The rotation transformation is described as:

$$
A_{\theta}=\left[\begin{array}{cc}
\cos \theta & -\sin \theta \\
\sin \theta & \cos \theta
\end{array}\right], 0 \leq \theta \leq 2 \pi
$$

According to the properties of affine transformation, it is easily concluded that any affine transformation can be expressed as a product of several above single-parameter linear transformations:

$$
A=A_{\theta} A_{n} A_{t} A_{s}=\left[\begin{array}{ll}
s \cos \theta & s t n \cos \theta-s t \sin \theta \\
s \sin \theta & s t n \sin \theta+s t \cos \theta
\end{array}\right]
$$

\section{Construction of affine moment invariants}

According to the model of affine transformation in section 2.2, the moment function $F$ will be defined as affine moment invariant, if $F$ has the invariant property on condition of translation, scale, slant and rotation transformation. Assuming that image $f(x, y)$ be expressed as $f_{T}\left(x^{\prime}, y^{\prime}\right)$ through affine transformation, then the centroid of the image $f_{T}\left(x^{\prime}, y^{\prime}\right)$ follows as:

$$
\left[\begin{array}{l}
\bar{x}^{\prime} \\
\bar{y}^{\prime}
\end{array}\right]=\left[\begin{array}{ll}
a_{11} & a_{12} \\
a_{21} & a_{22}
\end{array}\right]\left[\begin{array}{l}
\bar{x} \\
\bar{y}
\end{array}\right]+\left[\begin{array}{l}
b_{1} \\
b_{2}
\end{array}\right]
$$

Let formula (5) subtract formula (11), we can get the following formula:

$$
\left[\begin{array}{c}
x^{\prime}-\bar{x}^{\prime} \\
y^{\prime}-\bar{y}^{\prime}
\end{array}\right]=\left[\begin{array}{ll}
a_{11} & a_{12} \\
a_{21} & a_{22}
\end{array}\right]\left[\begin{array}{l}
x-\bar{x} \\
y-\bar{y}
\end{array}\right]
$$

Formula (14) shows that translation parameters $b_{1}$ and $b_{2}$ can be eliminated.

It can be proved that the moment function $F$ which remained unchanged through the affine transformation must be a invariant moment. Therefore, affine moment invariants can be derived according to the single-parameter linear transformations. Obviously, $F$ can be constructed by central moments, which has the form of polynomial on central moments, in order that the cancellation of affine transformation matrix A eliminate the distortion caused by the translation, scale, slant and rotation transformation.

There are several useful methods for construction of affine moment invariants, such as the apolar polynomial, the discriminant of polynomial and the Hankel determinant. This paper considers six affine moments as features of target image, which are constructed by less than four-order central moments[6].

$$
\begin{gathered}
F_{1}=2\left(u_{20} u_{02}-u_{11}^{2}\right) / u_{00}^{4} \\
F_{2}=\left(u_{30}^{2} u_{03}^{2}-6 u_{30} u_{21} u_{12} u_{03}+4 u_{30} u_{12}^{3}+4 u_{21}^{3} u_{03}-3 u_{12}^{2} u_{21}^{2}\right) / u_{00}^{10} \\
F_{3}=\left[u_{20}\left(u_{21} u_{03}-u_{12}^{2}\right)-u_{11}\left(u_{30} u_{03}-u_{21} u_{12}\right)\right. \\
\left.+u_{02}\left(u_{30} u_{12}-u_{21}^{2}\right)\right] / u_{00}^{7} \\
F_{4}=\left(u_{20}^{3} u_{03}^{2}-6 u_{20}^{2} u_{11} u_{12} u_{03}-6 u_{20}^{2} u_{02} u_{21} u_{03}+9 u_{20}^{2} u_{02} u_{12}^{2}\right. \\
+12 u_{20} u_{11}^{2} u_{21} u_{03}+6 u_{20} u_{11} u_{02} u_{30} u_{03}-18 u_{20} u_{11} u_{02} u_{21} u_{12} \\
-8 u_{11}^{3} u_{03} u_{30}-6 u_{20} u_{02}^{2} u_{30} u_{12}+9 u_{20} u_{02}^{2} u_{21}^{2} \\
\left.+12 u_{11}^{2} u_{02} u_{30} u_{12}-6 u_{11} u_{02}^{2} u_{30} u_{21}+u_{02}^{3} u_{30}^{2}\right) / u_{00}^{11} \\
F_{5}=2\left(u_{40} u_{04}-4 u_{13} u_{31}+3 u_{22}^{2}\right) / u_{00}^{6} \\
F_{6}=\left(u_{04} u_{22} u_{40}+2 u_{13} u_{22} u_{31}-u_{04} u_{31}^{2}-u_{40} u_{13}^{2}-u_{22}^{3}\right) / u_{00}^{9}
\end{gathered}
$$




\section{TARGET CLASSIFICATION AND RECOGNITION}

\section{EXPERIMENT ON AFFINE INVARIANT MOMENTS}

\section{A. Gray-scale image of space targets}

Gray-scale image sequences of four-class space targets are obtained with STK(Satellite Tool Kit)[7]. The parameters of four space targets follows as table 1.

\begin{tabular}{cccc}
\multicolumn{4}{c}{ TABLE1. PARAMETERS OF SIMULATED TARGETS } \\
\hline Class & Number & inclination & Semi-axis $(\mathrm{km})$ \\
& & angle $\left(^{\circ}\right)$ & \\
\hline Target 1 & 08026A & 98.803 & 7205 \\
Target 2 & 04018A & 98.910 & 7269 \\
Target 3 & 01047A & 97.173 & 6824 \\
Target 4 & 99020A & 98.184 & 7081
\end{tabular}

Gray-scale images of four-class simulated targets are showed as figure 1 .

\section{B. Targets classification and recognition experiment}

Four-class target image sequences are simulated in section 3.1 and training samples for each class targets are selected. Then, the affine invariant moments of the training samples for each class will be calculated, which compose an invariant moment vector. Target classification and recognition can be processed by calculating its affine invariant moments and matching with the invariant moment vectors of each class targets. The experiment procedure can be completed as the following steps:

Step 1: Select sample gray-scale images of four-class targets, considering those target images on condition of different gestures and different viewpoint angles.

Step 2: Calculate the affine invariant moments of sample images for each class target and construct the feature matrix composed of invariant moment vectors for each class target.

Step 3: Calculate the affine invariant moments of a target image to be recognized and match them with the feature matrix of each class target. The target will be considered to belong to the class, with which class the matching coefficient is maximum.

Targets classification and recognition experiments are simulated. The selected total of the four-class targets is 189,297,407 and 164 individually. According to affine invariant moments of each class target, the feature matrix is established. Different class and different total targets recognition experiments are completed and table 2 shows the recognition results that the ratio of correct recognition is higher than $82 \%$. The simulated result validates that target recognition based on affine invariant moments is effective.
TABLE.2 RECOGNITION RESULTS FOR FOUR-CLASS TARGETS BASED ON

\begin{tabular}{cccc}
\multicolumn{4}{c}{ AFFINE INVARIANT MOMENTS } \\
\hline Class & Total of & Total of correct & Ratio of correct \\
& target images & recognition & recognition (\%) \\
\hline Target 1 & 1697 & 1401 & 82.6 \\
Target 2 & 2665 & 2433 & 91.3 \\
Target 3 & 3661 & 3112 & 85.0 \\
Target 4 & 1474 & 1378 & 93.5 \\
\hline
\end{tabular}

\section{SUMMARIES}

An affine transformation model is described and analyzed in this paper. The affine transformation is decomposed into four basic single-parameter linear transformations, which are translation, scale, slant and rotation transformations. Six affine invariant moments are constructed with the central moments of digital gray-scale target images. Classification and recognition experiments for digital gray-scale images of four class targets are completed based on affine invariant moments and recognition results verifies its effectiveness. However, the gray-scale target images used in this paper are ideal, without any noise. In future work, noisy images and recognition methods may be considered as emphasis of our research.

\section{REFERENCES}

[1] Hu Ming-Kuei. Visual pattern recognition by moment invariants. IRE Trans Information Theory, 1962, 179-187.

[2] BI Xiao-jun, LIN Hong-zhang. Improvement of target identification algorithm for sequence image $[\mathrm{J}]$.Computer engineering. 2010,36(3):206-209.

[3] DU Ya-juan, ZHANG Hong-cai, PAN Quan. Three-Dimensional aircraft recognition using moments[J].Journal of Data Acquisition \&Processing. 2000,15(3):391-395.

[4] SHI Si-qi, SHI Guang-min, CHEN Xu-yang, LI Fu. Object recognition based on smoothing segmented-contour[J].ACTA PHOTONICA SINICA. 2010,39(5):929-934.

[5] ZHANG Hong-yue, YANG Xue-qin, GUO Hong-tao. Aircraft image recognition based on affine transformation[J].ACTA AERONAUTICA ET ASTRONAUTICA SINICA. 2003,24(3):251-254.

[6] LIU Yis-hu. Moment invariants in pattern recognition[D]. Guangzhou:Sun Yatsen University. 2002,31-43.

[7] YANG Ying, WANG qi. STK applied in computer simulation. National Defense Industry Publishing House. 2005,69-84 

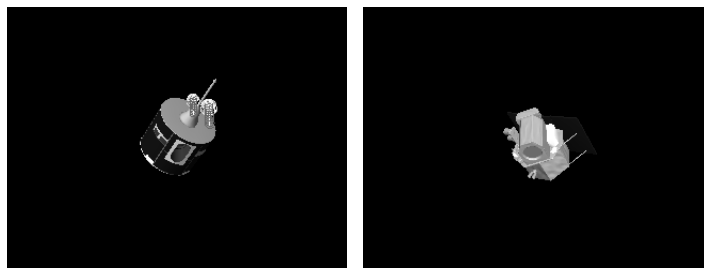

(a) Target 1

(b) Target 2

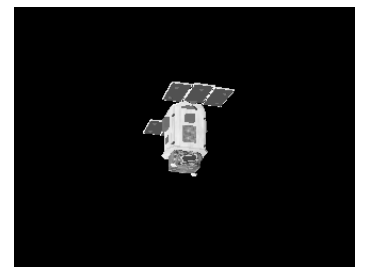

(c) Target 3

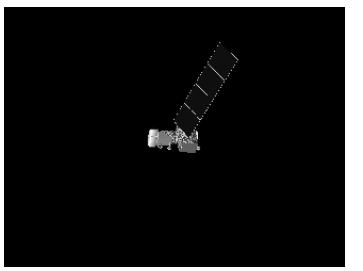

(d) Target 4

Figue 1. Gray-scale images of four-class simulated targets 